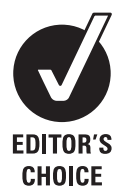

CHOICE

\title{
Patient-to-nurse ratio in neonatal ICU associated with daily weight gain, but not other clinical outcomes in moderately preterm infants
}

\section{Janet Tucker}

$10.1136 /$ ebn1070

University of Aberdeen, Aberdeen, Scotland

Correspondence to: Janet Tucker

Obstetrics and Gynaecology/ HSRU, Division of Applied Health Sciences, School of Medicine \& Dentistry, University of Aberdeen, Aberdeen Maternity Hospital, Cornhill Road, Aberdeen AB25 2ZL, Scotland; j.s.tucker@abdn.ac.uk

\section{Commentary on: Profit J, Petersen LA, McCormick MC, et al. Patient-to-nurse ratios and outcomes of moderately preterm infants. Pediatrics 2010;125:320-6.}

Reports from critical care settings more than a decade ago suggested poorer clinical outcomes were associated with lower nurse staffıng, but subsequent findings from studies in neonatal intensive care units (NICUs) have been more equivocal. Profit and colleagues have now examined outcomes for a prospective cohort of 850 moderately preterm infants (gestational age of $30-34^{6} / 7$ weeks) in relation to registered nurses on shift in 10 NICUs.

The measure of nursing provision used was the patient-nurse ratio (PNR). This was the average number of registered nurses (calculated over a $24 \mathrm{~h}$ period of shifts, including partial nurse shifts) and occupancy in terms of NICU census of midnight the previous day.

In addition to testing some infrequent clinical outcomes for this particular patient group and sample size (e.g. intraventricular haemorrhage and chronic lung disease), ${ }^{1}$ nosocomial infection and further proxy-outcome indicators were also tested. These indicators may be judged relevant and more sensitive indicators of nursing process and quality. They included measures of daily weight gain, gestational age at discharge, and any breast milk at discharge. Further follow-up information for the cohort was also available about subsequent acute emergency visits or rehospitalisation in the 3 months postdischarge.

A rudimentary comparison between smaller units and larger units revealed the following: the PNRs in large units were higher; their infants had longer stays and later gestational age at discharge, higher daily weight gain, more days of respiratory support and lower rates of any breast milk at discharge.

Multivariate linear and logistic regression models were used to adjust for patient-level characteristics that might also determine outcomes (such as gestation at birth, birth weight, gender and race/ethnicity as well as SNAP-II scores illness severity) and for the NICU-level characteristic of size and unit effects using general estimating equations. Sensitivity analyses were undertaken to both assess the impact of transfers and test outcomes for only a subset of the most ill infants in this defined patient population. Notably, after taking account of the infant-level and hospital-level factors, patient-nurse ratios (PNRs) alone were not reliably associated with any of the outcomes tested in this study.

\section{Challenges in this area of research}

Much of the previous evidence that has shown correlations of higher registered-nurse provision with improved clinical outcomes has come from studies that used administrative establishment-level data in large samples of hospitals, ${ }^{1}$ but findings are broad and descriptive, and causality cannot be inferred. In this study, Profit and colleagues explored exposures of infants to averaged, shiftby-shift, registered-nursing provision, adjusted for infant case-mix and illness severity, and included outcome indicators relevant to assessing nursing quality.

The authors acknowledge limitations of their study. The MPIP (Moderately Preterm Infants Project) ${ }^{2}$ took place in only 10 selected NICUs in California and Massachusetts between 2001 and 2003, so the generalisability of these findings and whether they would be replicated in contemporary practice is uncertain. The authors highlight the relatively small proportions of severely ill infants in this selected population and suggest that the study may lack statistical power to detect relatively rare events. Even the sensitivity analysis of their sub-set of infants in the upper quartile of severity (SNAP-II score >9) showed no independent association between any of the outcomes and PNR.

Using the PNR of registered nurses alone as the nursing provision measure is also a shortcoming. There is no further estimate of skillmix, including expert practitioners or aides, and no account of the composition of the wider care team. This includes medical staffing and many other hospital or unit-level factors that may contribute to drive quality of care and influence outcomes.

\section{What it means for practice and research}

The authors are cautious when interpreting the finding that lower PNRs in smaller units are not independently associated with better outcomes. They suggest this may not reflect excess staffing and that it may arise due to other factors. For example, fluctuations in occupancy per nurse are proportionately larger in smaller units, or RN role may be different with less ancillary support staff, compared to those in large units. Nursing staff play a central role in ensuring quality and improving patient care, but there still remains little evidence that increased numbers of registered nurses alone result in improved nursing outcomes for patients, as shown in a recent large study that involved analysing patient well-being both pre- and post-regulation change. ${ }^{3}$ Further information is needed about the impact and interplay of variation in nursing roles and skill-levels in clinical systems and organizations that may be undergoing many other 
concurrent changes and often already highly regulated with only relatively narrow variation in PNRs.

\section{Competing interests None.}

\section{References}

1. Kane RL, Shamliyan TA, Mueller C, et al. The association of registered nurse staffing levels and patient outcomes: systematic review and meta-analysis. Med Care 2007;45:1195-204.
2. Escobar GJ, McCormick MC, Zupancic JAF, et al. Unstudied infants: outcomes of moderately premature infants in the neonatal intensive care unit. Arch Dis Child Fetal Neonatal Ed 2006;91:F238-44.

3. Burnes Bolton L, Aydin CE, Donaldson N, et al. Mandated nurse staffing ratios in California: a comparison of staffing and nursing-sensitive outcomes pre- and postregulation. Policy Polit Nurs Pract 2007;8:238-50. 